\title{
Distributed Detection Fusion via Monte Carlo Importance Sampling
}

\author{
Hang Rao, Xiaojing Shen* Yunmin Zhu and Jianxin Pan
}

October 19, 2018

\begin{abstract}
Distributed detection fusion with high-dimension conditionally dependent observations is known to be a challenging problem. When a fusion rule is fixed, this paper attempts to make progress on this problem for the large sensor networks by proposing a new Monte Carlo framework. Through the Monte Carlo importance sampling, we derive a necessary condition for optimal sensor decision rules in the sense of minimizing the approximated Bayesian cost function. Then, a Gauss-Seidel/person-by-person optimization algorithm can be obtained to search the optimal sensor decision rules. It is proved that the discretized algorithm is finitely convergent. The complexity of the new algorithm is $O(L N)$ compared with $O\left(L N^{L}\right)$ of the previous algorithm where $L$ is the number of sensors and $N$ is a constant. Thus, the proposed methods allows us to design the large sensor networks with general high-dimension dependent observations. Furthermore, an interesting result is that, for the fixed AND or OR fusion rules, we can analytically derive the optimal solution in the sense of minimizing the approximated Bayesian cost function. In general, the solution of the Gauss-Seidel algorithm is only local optimal. However, in the new framework, we can prove that the solution of Gauss-Seidel algorithm is same as the analytically optimal solution in the case of the AND or OR fusion rule. The typical examples with dependent observations and large number of sensors are examined under this new framework. The results of numerical examples demonstrate the effectiveness of the new algorithm.
\end{abstract}

keywords: Distributed detection, Monte Carlo importance sampling, dependent observations, sensor decision rule, fusion rule

\section{Introduction}

Distributed signal detection has received significant attention in surveillance applications over the past thirty years [1, 2, 3, 4, 5, 6, 7, 8]. Tenney and Sandell [1] firstly considered Bayesian formulation of distributed

* This work was supported in part by the open research funds of BACC-STAFDL of China under Grant No. 2015afdl010, the special funds of NEDD of China under Grant No. 201314, the NSF No. 61273074 and the PCSIRT1273. Hang Rao, Xiaojing Shen (corresponding author), Yunmin Zhu and Jianxin Pan are with Department of Mathematics, Sichuan University, Chengdu, Sichuan 610064, China. E-mail: shenxj@scu.edu.cn,ymzhu@scu.edu.cn, jianxin.pan@manchester.ac.uk. 
detection for parallel sensor network structures and proved that the optimal decision rules at the sensors are likelihood ratio (LR) for conditionally independent sensor observations. However, the optimal thresholds of LR at individual sensors can be only obtained by solving a set of coupled nonlinear equations. When the sensor decision rules are fixed, Chair and Varshney [3] derived an optimal fusion rule based on the LR test. For conditionally independent sensor observations, many excellent results on distributed detection have been derived and are summarized in [4] and references therein. The emerging wireless sensor networks [7] motivated the optimality of LR thresholds to be extended to non-ideal detection systems in which sensor outputs are to be communicated through noisy, possibly coupled channels to the fusion center [6, 9, 10].

There is much less attention on the studies of sensor decision rules for generally dependent observations which were considered to be difficult (see, e.g., [1, 2, 11]). Tsitsiklis and Athans [2] provided a rigorous mathematical analysis to demonstrate the computational difficulty in obtaining the optimal sensor decision rules for dependent sensor observations. However, some progresses have been made for the special dependent observations cases (see, e.g., [12, 13, 14, 15, 16, 17, 18]). Willett et al. [18] discussed difficulties for dealing with dependent observations. Zhu et al.[19] proposed a computationally efficient iterative algorithm which computes a discrete approximation of the optimal sensor decision rules for general dependent observations and a fixed fusion rule. This algorithm converges in finite steps. In [20], the authors developed an efficient algorithm to simultaneously search for the optimal fusion rule and the optimal sensor rules by combining the methods of Chair and Varshney [3] and Zhu et al. [19]. Recently, a new framework for distributed detection with conditionally dependent observations was introduced in [21], which can identify several classes of problems with dependent observations whose optimal sensor decision rules resemble the ones for the independent case.

Although large sensor networks have attracted much attention in both theory and application [22, 23, 24], the studies of sensor decision rules for large sensor networks with general dependent observations have had little progress. The fundamental reason is that the computation complexity is $O\left(L N^{L}\right)$ for the previous algorithms, where $L$ is the number of sensors and $N$ is a given constant. In this paper, we propose a new Monte Carlo framework to overcome the limitation of the discretized algorithms in [19, 20] for the large sensor networks. Through the Monte Carlo importance sampling [25, 26], the Bayesian cost function is approximated by the sample average by the strong law of large number. Then, we derive a necessary condition for optimal sensor decision rules so that a Gauss-Seidel optimization algorithm can be obtained to search the optimal sensor decision rules. It is proved that the new discretized algorithm is finitely convergent. The complexity of the new algorithm is order of $O(L N)$ compared with $O\left(L N^{L}\right)$ of the algorithms in [19, 20]. Thus, the proposed methods allows us to design the large sensor networks with general dependent observations. Furthermore, an interesting result is that, for the fixed AND or OR fusion rules, we can analytically derive the optimal solution in the sense of minimizing the approximated Bayesian cost function. In general, the solution of the Gauss-Seidel algorithm is only local optimal. However, in the new framework, we can prove that the solution of Gauss-Seidel algorithm is same as the analytically optimal solution when the fusion rule is the AND or OR. The typical examples with dependent observations and large number of sensors are 
examined under this new framework. The results of numerical examples demonstrate the effectiveness of the new algorithm. The performance of the new algorithm based on Mixture-Gaussian trial distribution is better than that based on Gaussian trial distribution.

The rest of the paper is organized as follows. Preliminaries are given in Section 2, including problem formulation and Monte Carlo approximation of the cost function. In Section 3, necessary conditions for optimal sensor decision rules are given. In Section 4, a Gauss-Seidel iterative algorithm is presented based on the necessary conditions. The convergence of this algorithm is proved. For the fixed AND or OR fusion rules, the optimal solution in the sense of minimizing the approximated Bayesian cost function can be analytically derived. Moreover, we prove that the solution of Gauss-Seidel algorithm is same as the analytically optimal solution in the case of the AND or OR fusion rule. In Section 5, numerical examples are given that exhibit the effectiveness of the new algorithm class. In Section 6 , we draw conclusions.

\section{Preliminaries}

\subsection{Problem formulation}

The $L$-sensor Bayesian detection model with two hypotheses $H_{0}$ and $H_{1}$ are considered as follows. A parallel architecture is assumed. The $i$ th sensor compresses the $n_{i}$-dimensional vector observation $y_{i}$ to one bit: $I_{i}\left(y_{i}\right): \mathbb{R}^{n_{i}} \rightarrow\{0,1\}, i=1, \ldots, L$. In this paper, we consider deterministic (non-randomized) decision rules. When the fusion rule $F$ is fixed, the distributed multisensor Bayesian decision problem is to minimize the following Bayesian cost function by optimizing the sensor decision rule $I_{1}\left(y_{1}\right), \ldots, I_{L}\left(y_{L}\right)$,

$$
\begin{aligned}
& C\left(I_{1}\left(y_{1}\right), \ldots, I_{L}\left(y_{L}\right) ; F\right) \\
= & C_{00} P_{0} P\left(F=0 \mid H_{0}\right)+C_{01} P_{1} P\left(F=0 \mid H_{1}\right) \\
& +C_{10} P_{0} P\left(F=1 \mid H_{0}\right)+C_{11} P_{1} P\left(F=1 \mid H_{1}\right),
\end{aligned}
$$

where $C_{i j}$ are the known cost coefficients, $P_{0}$ and $P_{1}$ are the prior probabilities for the hypotheses $H_{0}$ and $H_{1}$, and $P\left(F=i \mid H_{j}\right)$ is the probability that the fusion center decides for hypothesis $i$ given hypothesis $H_{j}$ is true. The general form of the binary fusion rule $F$ is denoted by an indicator function on a set $S=$ $\left\{\left(u_{1}, \ldots, u_{L}\right): u_{i}=0 / 1, i=1,2, \ldots, L\right\}:$

$$
F\left(\left(u_{1}, \ldots, u_{L}\right)\right): S \rightarrow\{0,1\} .
$$

Note that a fusion rule is a binary division of the set $S$ and the number of elements of the set $S$ is $2^{L}$, thus there exists $2^{2^{L}}$ fusion rules. Let $s_{k}$ be the $k$-th element of $S, k=1, \ldots, 2^{L}$. Every $s_{k}$ is $L$-dimensional vector and $s_{k}(i)=0$ or $1, i=1, \ldots, L$. For convenience, we denote sets $S_{0}$ and $S_{1}$ as the elements in $S$ for which the algorithm took decision $H_{0}$ and $H_{1}$ respectively, i.e.

$$
\begin{aligned}
& S_{0}=\left\{s_{k}: F\left(s_{k}\right)=0, k=1, \ldots, 2^{L}\right\}, \\
& S_{1}=\left\{s_{k}: F\left(s_{k}\right)=1, k=1, \ldots, 2^{L}\right\} .
\end{aligned}
$$


Moreover, we let $\Omega=\mathbb{R}^{n_{1}} \times \ldots \times \mathbb{R}^{n_{L}}$ and denote

$$
\begin{gathered}
\Omega_{0}=\left\{\left(y_{1}, \ldots, y_{L}\right): I_{1}\left(y_{1}\right)=s_{k}(1), \ldots, I_{L}\left(y_{L}\right)=s_{k}(L) ;\right. \\
\left.F\left(s_{k}\right)=0, k=1, \ldots, 2^{L}\right\} \\
\Omega_{1}=\left\{\left(y_{1}, \ldots, y_{L}\right): I_{1}\left(y_{1}\right)=s_{k}(1), \ldots, I_{L}\left(y_{L}\right)=s_{k}(L) ;\right. \\
\left.F\left(s_{k}\right)=1, k=1, \ldots, 2^{L}\right\} .
\end{gathered}
$$

Obviously, $S=S_{0} \bigcup S_{1}$ and $\Omega=\Omega_{0} \bigcup \Omega_{1}$. Suppose that $p\left(y_{1}, y_{2}, \ldots, y_{L} \mid H_{1}\right)$ and $p\left(y_{1}, y_{2}, \ldots, y_{L} \mid H_{0}\right)$ are the known conditional joint probability density functions under each hypothesis.

Substituting the definitions of fusion rule $F$ and sensor decision rule $I_{i}\left(y_{i}\right)$ into (1) and simplifying, we have

$$
\begin{aligned}
& C\left(I_{1}\left(y_{1}\right), \ldots, I_{L}\left(y_{L}\right) ; F\right) \\
= & C_{10} P_{0}+C_{11} P_{1}+\int_{\Omega_{0}}\left\{\left[P_{1}\left(C_{01}-C_{11}\right) p\left(y_{1}, \ldots, y_{L} \mid H_{1}\right)\right]\right. \\
& \left.-\left[P_{0}\left(C_{10}-C_{00}\right) p\left(y_{1}, \ldots, y_{L} \mid H_{0}\right)\right]\right\} d y_{1} \cdots d y_{L} \\
= & c+\int_{\Omega} I_{\Omega_{0}}\left(y_{1}, \ldots, y_{L}\right) \hat{L}\left(y_{1}, \ldots, y_{L}\right) d y_{1} \ldots d y_{L}
\end{aligned}
$$

where $I_{\Omega_{0}}\left(y_{1}, \ldots, y_{L}\right)$ is an indicator function on $\Omega_{0}$,

$$
\begin{array}{r}
\hat{L}\left(y_{1}, \ldots, y_{L}\right)=a p\left(y_{1}, \ldots, y_{L} \mid H_{1}\right)-b p\left(y_{1}, \ldots, y_{L} \mid H_{0}\right), \\
a=P_{1}\left(C_{01}-C_{11}\right), \quad b=P_{0}\left(C_{10}-C_{00}\right), \quad c=C_{10} P_{0}+C_{11} P_{1} .
\end{array}
$$

$a, b, c$ are fixed constants.

The indicator function $I_{\Omega_{0}}\left(y_{1}, \ldots, y_{L}\right)$ can be written as $L$ equivalent polynomials of the sensor decision rules $I_{1}\left(y_{1}\right), \ldots, I_{L}\left(y_{L}\right)$ and the fusion rule $F$ as follows (see [20]):

$$
\begin{aligned}
I_{\Omega_{0}}\left(y_{1}, \ldots, y_{L}\right)= & {\left[1-I_{1}\left(y_{1}\right)\right] P_{11}\left(I_{2}\left(y_{2}\right), \ldots, I_{L}\left(y_{L}\right) ; F\right) } \\
& +P_{12}\left(I_{2}\left(y_{2}\right), \ldots, I_{L}\left(y_{L}\right) ; F\right) \\
& \ldots \ldots \\
= & {\left[1-I_{L}\left(y_{L}\right)\right] P_{L 1}\left(I_{1}\left(y_{1}\right), I_{2}\left(y_{2}\right), \ldots, I_{L-1}\left(y_{L-1}\right) ; F\right) } \\
& +P_{L 2}\left(I_{1}\left(y_{1}\right), I_{2}\left(y_{2}\right), \ldots, I_{L-1}\left(y_{L-1}\right) ; F\right)
\end{aligned}
$$


where, for $j=1, \ldots, L$,

$$
\begin{aligned}
& P_{j 1}\left(I_{1}\left(y_{1}\right), \ldots, I_{j-1}\left(y_{j-1}\right), I_{j+1}\left(y_{j+1}\right), \ldots, I_{L}\left(y_{L}\right) ; F\right) \\
\triangleq & \sum_{k=1}^{2^{L}}\left\{\left[1-F\left(s_{k}\right)\right]\left[1-2 s_{k}(j)\right] \prod_{m=1, m \neq j}^{2^{L}}\left[s_{k}(m) I_{m}\left(y_{m}\right)+\left(1-s_{k}(m)\right)\left(1-I_{m}\left(y_{m}\right)\right)\right]\right\} \\
& P_{j 2}\left(I_{1}\left(y_{1}\right), \ldots, I_{j-1}\left(y_{j-1}\right), I_{j+1}\left(y_{j+1}\right), \ldots, I_{L}\left(y_{L}\right) ; F\right) \\
\triangleq & \sum_{k=1}^{2^{L}}\left\{\left[1-F\left(s_{k}\right)\right] s_{k}(j) \prod_{m=1, m \neq j}^{2^{L}}\left[s_{k}(m) I_{m}\left(y_{m}\right)+\left(1-s_{k}(m)\right)\left(1-I_{m}\left(y_{m}\right)\right)\right]\right\}
\end{aligned}
$$

Note that both $P_{j 1}\left(I_{1}\left(y_{1}\right), \ldots, I_{j-1}\left(y_{j-1}\right), I_{j+1}\left(y_{j+1}\right), \ldots, I_{L}\left(y_{L}\right) ; F\right)$ and $P_{j 2}\left(I_{1}\left(y_{1}\right), \ldots, I_{j-1}\left(y_{j-1}\right)\right.$, $\left.I_{j+1}\left(y_{j+1}\right), \ldots, I_{L}\left(y_{L}\right) ; F\right)$ are independent of $I_{j}\left(y_{j}\right)$ for $j=1, \ldots, L$. For convenience, we also denote them by $P_{j 1}(\cdot), P_{j 2}(\cdot)$, respectively. Moreover, (12) is also a key equation in the following results.

\subsection{Monte Carlo importance sampling}

In this section, we present an approximation of the cost function (7) by Monte Carlo importance sampling (see, e.g., [25, 26]). More specifically, assume that the samples $Y_{1}, \ldots, Y_{N}$ are from population $Y$ with a given trial distribution $g\left(y_{1}, y_{2}, \ldots, y_{L}\right)$, where $Y_{i}=\left[Y_{1 i}, Y_{2 i}, \ldots, Y_{L i}\right]^{T}$. From (7),

$$
\begin{aligned}
& C\left(I_{1}\left(y_{1}\right), \ldots, I_{L}\left(y_{L}\right) ; F\right) \\
= & \int_{\Omega} \frac{I_{\Omega_{0}}\left(y_{1}, y_{2}, \ldots, y_{L}\right) \hat{L}\left(y_{1}, y_{2}, \ldots, y_{L}\right) g\left(y_{1}, y_{2}, \ldots, y_{L}\right)}{g\left(y_{1}, y_{2}, \ldots, y_{L}\right)} d y_{1} \ldots d y_{L}+c \\
= & \mathbb{E}_{g} \frac{I_{\Omega_{0}}(Y) \hat{L}(Y)}{g(Y)}+c \\
\approx & \frac{1}{N} \sum_{i=1}^{N} \frac{I_{\Omega_{0}}\left(Y_{1 i}, Y_{2 i}, \ldots, Y_{L i}\right) \hat{L}\left(Y_{1 i}, Y_{2 i}, \ldots, Y_{L i}\right)}{g\left(Y_{1 i}, Y_{2 i}, \ldots, Y_{L i}\right)}+c \\
\triangleq & C_{M C}\left(I_{1}\left(y_{1}\right), \ldots, I_{L}\left(y_{L}\right) ; F, N\right),
\end{aligned}
$$

where $g\left(y_{1}, y_{2}, \ldots, y_{L}\right)$ is the trial density such that (14) is well-defined. (15) is from $Y \sim g\left(y_{1}, y_{2}, \ldots, y_{L}\right)$. (16) is denoted by $C_{M C}\left(I_{1}\left(y_{1}\right), \ldots, I_{L}\left(y_{L}\right) ; F, N\right)$. Based on the strong law of large number, (15) can be approximated by (16), i.e., $C_{M C}\left(I_{1}\left(y_{1}\right), \ldots, I_{L}\left(y_{L}\right) ; F, N\right) \rightarrow C\left(I_{1}\left(y_{1}\right), \ldots, I_{L}\left(y_{L}\right) ; F\right)$, a.s. as $N \rightarrow$

$\infty$. The optimal trial distribution is $g\left(y_{1}, y_{2}, \ldots, y_{L}\right) \propto\left|I_{\Omega_{0}}\left(y_{1}, y_{2}, \ldots, y_{L}\right) \hat{L}\left(y_{1}, y_{2}, \ldots, y_{L}\right)\right|$ (see, e.g., [25, 26]). By (10), (11) and (16), so that we have 


$$
\begin{aligned}
& C_{M C}\left(I_{1}\left(y_{1}\right), \ldots, I_{L}\left(y_{L}\right) ; F, N\right) \\
= & \frac{1}{N} \sum_{i=1}^{N} \frac{\left[1-I_{1}\left(Y_{1 i}\right)\right] P_{11}\left(I_{2}\left(Y_{2 i}\right), \ldots, I_{L}\left(Y_{L i}\right) ; F\right) \hat{L}\left(Y_{1 i}, Y_{2 i}, \ldots, Y_{L i}\right)}{g\left(Y_{1 i}, Y_{2 i}, \ldots, Y_{L i}\right)} \\
& +\frac{1}{N} \sum_{i=1}^{N} \frac{P_{12}\left(I_{2}\left(Y_{2 i}\right), \ldots, I_{L}\left(Y_{L i}\right) ; F\right) \hat{L}\left(Y_{1 i}, Y_{2 i}, \ldots, Y_{L i}\right)}{g\left(Y_{1 i}, Y_{2 i}, \ldots, Y_{L i}\right)}+c \\
& \ldots \ldots \\
= & \frac{1}{N} \sum_{i=1}^{N} \frac{\left[1-I_{L}\left(Y_{L i}\right)\right] P_{L 1}\left(I_{1}\left(Y_{1 i}\right), \ldots, I_{L-1}\left(Y_{(L-1) i}\right) ; F\right) \hat{L}\left(Y_{1 i}, Y_{2 i}, \ldots, Y_{L i}\right)}{g\left(Y_{1 i}, Y_{2 i}, \ldots, Y_{L i}\right)} \\
& +\frac{1}{N} \sum_{i=1}^{N} \frac{P_{L 2}\left(I_{1}\left(Y_{1 i}\right), \ldots, I_{L-1}\left(Y_{(L-1) i}\right) ; F\right) \hat{L}\left(Y_{1 i}, Y_{2 i}, \ldots, Y_{L i}\right)}{g\left(Y_{1 i}, Y_{2 i}, \ldots, Y_{L i}\right)}+c
\end{aligned}
$$

\section{Necessary Conditions For Optimum Sensor Decision Rules}

The distributed detection fusion problem is to minimize the Bayesian cost function $C\left(I_{1}\left(y_{1}\right), \ldots, I_{L}\left(y_{L}\right)\right.$; F) (7). Based on the Monte Carlo approximation (17), we concentrate on selecting a set of optimal sensor decision rules $I_{1}\left(y_{1}\right), \ldots, I_{L}\left(y_{L}\right)$ such that the approximated cost function $C_{M C}\left(I_{1}\left(y_{1}\right), \ldots, I_{L}\left(y_{L}\right) ; F, N\right)$ is minimum.

Firstly, we prove that the minimum of the $C_{M C}\left(I_{1}\left(y_{1}\right), \ldots, I_{L}\left(y_{L}\right) ; F, N\right)$ cost functional converges to the infimum of the cost function $C\left(I_{1}, \ldots, I_{L} ; F\right)$ as the sample size $N$ tends to infinity, under some mild assumptions. Since the deterministic (non-randomized) decision rules are considered in this paper, in the following sections, we assume that the samples drawn from the trial distribution have been fixed so that $C_{M C}\left(I_{1}\left(y_{1}\right), \ldots, I_{L}\left(y_{L}\right) ; F, N\right)$ has no randomness.

Theorem 3.1. Let $C_{\text {inf }}$ be the infimum of $C\left(I_{1}, \ldots, I_{L} ; F\right)$ and $C_{M C}^{*}\left(I_{1}, \ldots, I_{L} ; F, N\right)$ be the minimum of the Monte Carlo approximation $C_{M C}\left(I_{1}, \ldots, I_{L} ; F, N\right)$ (17) where $I_{1}, \ldots, I_{L}$ are decision variables. If $C_{M C}\left(I_{1}, \ldots, I_{L} ; F, N\right)$ satisfies

$$
\left|C\left(I_{1}, \ldots, I_{L} ; F\right)-C_{M C}\left(I_{1}, \ldots, I_{L} ; F, N\right)\right|<\frac{\delta}{\sqrt{N}},
$$

where the constant $\delta$ does not depend on $I_{1}, \ldots, I_{L}, F$ and $N$, then we have

$$
\lim _{N \rightarrow \infty} C_{M C}^{*}\left(I_{1}, \ldots, I_{L} ; F, N\right)=\inf _{I_{1}, \cdots, I_{L}} C\left(I_{1}, \ldots, I_{L} ; F\right) \triangleq C_{\text {inf }}
$$

Proof. By the definition of $C_{\text {inf }}$, for arbitrary $\epsilon>0$, there exists a set of sensor rules $\left(I_{1}, \ldots, I_{L}\right)$ such that 


$$
C\left(I_{1}, \ldots, I_{L} ; F\right) \leq C_{\text {inf }}+\frac{1}{2} \epsilon
$$

Since definition of $C_{M C}\left(I_{1}, \ldots, I_{L} ; F, N\right)$ and (20), there exists $N^{*}=\left(\frac{2 \delta}{\epsilon}\right)^{2}>0$ such that for any $N \geq N^{*}$

$$
C_{M C}\left(I_{1}, \ldots, I_{L} ; F, N\right) \leq C\left(I_{1}, \ldots, I_{L} ; F\right)+\frac{1}{2} \epsilon .
$$

Thus, $C_{M C}\left(I_{1}, \ldots, I_{L} ; F, N\right) \leq C_{i n f}+\epsilon$. By the definition of $C_{M C}^{*}\left(I_{1}, \ldots, I_{L} ; F, N\right)$, we have

$$
C_{M C}^{*}\left(I_{1}, \ldots, I_{L} ; F, N\right) \leq C_{M C}\left(I_{1}, \ldots, I_{L} ; F, N\right) \leq C_{i n f}+\epsilon \text { for } \forall N \geq N^{*},
$$

which implies that

$$
\lim \sup _{N \rightarrow \infty} C_{M C}^{*}\left(I_{1}, \ldots, I_{L} ; F, N\right) \leq C_{i n f}+\epsilon
$$

Since $\epsilon$ is arbitrary, we have

$$
\lim \sup _{N \rightarrow \infty} C_{M C}^{*}\left(I_{1}, \ldots, I_{L} ; F, N\right) \leq C_{\text {inf }} .
$$

On the other hand, suppose that

$$
\lim \inf _{N \rightarrow \infty} C_{M C}^{*}\left(I_{1}, \ldots, I_{L} ; F, N\right)<C_{\text {inf }} .
$$

Then there would be a positive constant $\tau>0$, and a sequence $\left\{N_{k}\right\}$ such that $N_{k} \rightarrow \infty$, and

$$
C_{M C}^{*}\left(I_{1}, \ldots, I_{L} ; F, N_{k}\right)<C_{\text {inf }}-\tau \text {. }
$$

For every such $C_{M C}^{*}\left(I_{1}, \ldots, I_{L} ; F, N_{k}\right)$, there must be a set of $\left(I_{1}^{k}, I_{2}^{k} \ldots, I_{L}^{k}\right)$ such that

$$
C_{M C}^{*}\left(I_{1}, \ldots, I_{L} ; F, N_{k}\right)=C_{M C}\left(I_{1}^{k}, I_{2}^{k} \ldots, I_{L}^{k} ; F, N_{k}\right)
$$

Using the inequality (20) and (23), for large enough $K$, we have $\frac{\delta}{\sqrt{N_{K}}}<\tau$,

$$
C\left(I_{1}^{K}, I_{2}^{K} \ldots, I_{L}^{K} ; F\right) \leq C_{M C}^{*}\left(I_{1}, \ldots, I_{L} ; F, N_{K}\right)+\tau<C_{i n f},
$$

which contradicts the definition of $C_{i n f}$. Therefore,

$$
\lim \inf _{N \rightarrow \infty} C_{M C}^{*}\left(I_{1}, \ldots, I_{L} ; F, N\right) \geq C_{\text {inf }} .
$$

By the inequality (22) and (24),

$$
C_{i n f} \leq \lim \inf _{N \rightarrow \infty} C_{M C}^{*}\left(I_{1}, \ldots, I_{L} ; F, N\right) \leq \lim \sup _{N \rightarrow \infty} C_{M C}^{*}\left(I_{1}, \ldots, I_{L} ; F, N\right) \leq C_{i n f},
$$

which implies that (21). 
Remark 3.2. The assumption (20) is not restrictive, since, by the central limit theorem, the error term of this Monte Carlo approximation is $O\left(N^{-1 / 2}\right)$ regardless of the dimensionality of $Y$ (see [25]).

Secondly, we derive the necessary conditions for optimal sensor decision rules in the sense of minimizing $C_{M C}\left(I_{1}\left(y_{1}\right), \ldots, I_{L}\left(y_{L}\right) ; F, N\right)$ for a parallel distributed detection system.

Theorem 3.3. If $\left\{I_{1}\left(y_{1}\right), \ldots, I_{L}\left(y_{L}\right)\right\}$ are a set of optimal sensor decision rules which minimize $C_{M C}\left(I_{1}\left(y_{1}\right)\right.$, $\left.\ldots, I_{L}\left(y_{L}\right) ; F, N\right)$ in (16) in a parallel distributed Bayesian detection fusion system, then $\left\{I_{1}\left(y_{1}\right), \ldots, I_{L}\left(y_{L}\right)\right\}$ must satisfy the following equations:

$$
\begin{aligned}
& I_{1}\left(Y_{1 i}\right)=I\left[P_{11}\left(I_{2}\left(Y_{2 i}\right), I_{3}\left(Y_{3 i}\right), \ldots, I_{L}\left(Y_{L i}\right) ; F\right)\right. \\
& \left.\cdot \hat{L}\left(Y_{1 i}, Y_{2 i}, \ldots, Y_{L i}\right)\right], \quad \text { for } i=1, \ldots, N \\
& I_{2}\left(Y_{2 i}\right)=I\left[P_{21}\left(I_{1}\left(Y_{1 i}\right), I_{3}\left(Y_{3 i}\right), \ldots, I_{L}\left(Y_{L i}\right) ; F\right)\right. \\
& \left.\cdot \hat{L}\left(Y_{1 i}, Y_{2 i}, \ldots, Y_{L i}\right)\right], \quad \text { for } i=1, \ldots, N \\
& I_{L}\left(Y_{L i}\right)=I\left[P_{L 1}\left(I_{1}\left(Y_{1 i}\right),\left(I_{2}\left(Y_{2 i}\right)\right), \ldots, I_{L-1}\left(Y_{(L-1) i}\right) ; F\right)\right. \\
& \text {. } \left.\hat{L}\left(Y_{1 i}, Y_{2 i}, \ldots, Y_{L i}\right)\right], \quad \text { for } i=1, \ldots, N
\end{aligned}
$$

where $P_{j 1}(\cdot), j=1, \ldots, L$ are defined by $([12), I[\cdot]$ is an indicator function denoted as follows:

$$
I[x]= \begin{cases}1, & \text { if } x \geq 0 \\ 0, & \text { if } x<0\end{cases}
$$

Proof. Since both $P_{j 1}(\cdot)$ and $P_{j 2}(\cdot)$ are independent of $I_{j}\left(y_{j}\right)$ for $j=1, \ldots, L$, if $I_{1}\left(y_{1}\right)$ minimizes the Monte Carlo approximation of (16), then $I_{1}\left(Y_{1 i}\right)$ should be equal to 1 when $P_{11}\left(I_{2}\left(Y_{2 i}\right), I_{3}\left(Y_{3 i}\right), \ldots, I_{L}\left(Y_{L i}\right)\right.$; $F) \hat{L}\left(Y_{1 i}, Y_{2 i}, \ldots, Y_{L i}\right)$ is positive for $i=1, \ldots, N$, otherwise it should be equal to 0 . Thus, we have (25) by the definition of $I[x]$ in (28). Similarly, by (19), we have (26)-(27).

\section{Monte Carlo Gauss-Seidel Iterative Algorithm Its Convergence}

\subsection{Monte Carlo Gauss-Seidel Iterative Algorithm}

Let the sensor decision rules at the $k$ th stage of iteration be denoted by $\left\{\left(I_{1}^{k}\left(Y_{1 i}\right), \ldots, I_{L}^{k}\left(Y_{L i}\right)\right), i=1, \ldots, N\right\}$ with the initial set $\left\{\left(I_{1}^{0}\left(Y_{1 i}\right), \ldots, I_{L}^{0}\left(Y_{L i}\right)\right), i=1, \ldots, N\right\}$. Suppose the fusion rule is fixed. Based on Theorem 3.3. we can drive a Gauss-Seidel iterative algorithm for minimizing $C_{M C}\left(I_{1}\left(y_{1}\right), \ldots, I_{L}\left(y_{L}\right) ; F, N\right)$ in (17) as follows.

Algorithm 4.1 (Monte Carlo Gauss-Seidel iterative algorithm). 
- Step 1: Draw samples $Y_{1}, \ldots, Y_{N}$ from an importance density $g\left(y_{1}, y_{2}, \ldots, y_{L}\right)$.

- Step 2: Given a fusion rule $F$ and initialize $L$ sensor decision rules $j=1, \ldots, L$,

$$
I_{j}^{0}\left(Y_{j i}\right)=0 / 1 \quad \text { for } \quad i=1, \ldots, N
$$

- Step 3: Iteratively search L sensor decision rules for better system performance until a terminate criterion step 4 is satisfied. The $(k+1)$ th stage of the iteration is as follows:

$$
\begin{aligned}
& I_{1}^{k+1}\left(Y_{1 i}\right)=I\left[P_{11}\left(I_{2}^{k}\left(Y_{2 i}\right), I_{3}^{k}\left(Y_{3 i}\right), \ldots, I_{L}^{k}\left(Y_{L i}\right) ; F\right)\right. \\
& \left.\cdot \hat{L}\left(Y_{1 i}, Y_{2 i}, \ldots, Y_{L i}\right)\right], \quad \text { for } i=1, \ldots, N \\
& I_{2}^{k+1}\left(Y_{2 i}\right)=I\left[P_{21}\left(I_{1}^{k+1}\left(Y_{2 i}\right), I_{3}^{k}\left(Y_{3 i}\right), \ldots, I_{L}^{k}\left(Y_{L i}\right) ; F\right)\right. \\
& \left.\cdot \hat{L}\left(Y_{1 i}, Y_{2 i}, \ldots, Y_{L i}\right)\right], \quad \text { for } i=1, \ldots, N \\
& I_{L}^{k+1}\left(Y_{L i}\right)=I\left[P_{L 1}\left(I_{1}^{k+1}\left(Y_{1 i}\right),\left(I_{2}^{k+1}\left(Y_{2 i}\right)\right), \ldots, I_{L-1}^{k+1}\left(Y_{(L-1) i}\right) ; F\right)\right. \\
& \text { - } \left.\hat{L}\left(Y_{1 i}, Y_{2 i}, \ldots, Y_{L i}\right)\right], \quad \text { for } i=1, \ldots, N \text {. }
\end{aligned}
$$

- Step 4: A termination criterion of the iteration process is, for $i=1, \ldots, N$

$$
\begin{array}{rll}
I_{1}^{k+1}\left(Y_{1 i}\right) & = & I_{1}^{k}\left(Y_{1 i}\right) \\
I_{2}^{k+1}\left(Y_{2 i}\right) & = & I_{2}^{k}\left(Y_{2 i}\right), \\
& \ldots \cdots & \\
I_{L}^{k+1}\left(Y_{L i}\right) & = & I_{L}^{k}\left(Y_{L i}\right) .
\end{array}
$$

Remark 4.2. Once we obtain $I_{1}\left(Y_{1 i}\right)$ for $i=1, \ldots, N$, then $I_{1}(y)$ can be obtained by defining $I_{1}\left(y_{1}\right)=$ $I_{1}\left(Y_{1 i}\right)$ when the distance $\left\|y_{1}-Y_{1 i}\right\|$ is less than $\left\|y_{1}-Y_{1 j}\right\|$, for all $j \neq i$. Similarly, we can obtain $I_{i}\left(y_{i}\right)$ for $i=2, \ldots, L$.

Remark 4.3. The main computation burden of Algorithm 4.1 is in (30)-(32). If the number of discretized points $N_{1}=N_{2}=\ldots=N_{L}=N$ in (10) of [19], then $P_{j 1}(\cdot) \hat{L}\left(Y_{1 i}, Y_{2 i}, \ldots, Y_{L i}\right), j=1, \ldots, L, i=$ $1, \ldots, N$ are computed $L \times N$ times in Algorithm 4.1. However, in [19], they are computed $L N^{L}$ times. In next section, we prove Algorithm 4.1 terminates in finite steps. Thus, the computation complexity of Algorithm 4.1 is $O(L N)$ compared with $O\left(L N^{L}\right)$ of the algorithm in [19].

\subsection{Convergence of Monte Carlo Gauss-Seidel Iterative Algorithm}

Now we prove that Algorithm 4.1 must converge to a local optimal value and the algorithm cannot oscillate infinitely often, i.e., terminate after a finite number of iterations.

For convenience, for $j=1, \ldots, L$, we denote $C_{M C}(18)-(19)$ in the $(k+1)$ th iteration process by 


$$
\begin{aligned}
& C_{M C}\left(I_{1}^{k+1}, \ldots, I_{j}^{k+1}, I_{j+1}^{k}, \ldots, I_{L}^{k} ; F, N\right) \\
= & c+\frac{1}{N} \sum_{i=1}^{N}\left\{\left[1-I_{j}^{k+1}\left(Y_{j i}\right)\right] P_{j 1}\left(I_{1}^{k+1}\left(Y_{1 i}\right), \ldots, I_{j-1}^{k+1}\left(Y_{(j-1) i}\right), I_{j+1}^{k}\left(Y_{(j+1) i}\right), \ldots, I_{L}^{k}\left(Y_{L i}\right) ; F, N\right)\right. \\
& \left.+P_{j 2}\left(I_{1}^{k+1}\left(Y_{1 i}\right), \ldots, I_{j-1}^{k+1}\left(Y_{(j-1) i}\right), I_{j+1}^{k}\left(Y_{(j+1) i}\right), \ldots, I_{L}^{k}\left(Y_{L i}\right) ; F, N\right)\right\} \frac{\hat{L}\left(Y_{1 i}, Y_{2 i}, \ldots, Y_{L i}\right)}{g\left(Y_{1 i}, Y_{2 i}, \ldots, Y_{L i}\right)} .
\end{aligned}
$$

Similarly, we denote the $(k+1)$ th iteration process of the iterative items $P_{j 1}(\cdot) \hat{L}(\cdot)$ in (30)-(32) by

$$
\begin{aligned}
G_{j}^{k+1}\left(Y_{j i}\right)= & P_{j 1}\left(I_{1}^{k+1}\left(Y_{1 i}\right), \ldots, I_{j-1}^{k+1}\left(Y_{(j-1) i}\right), I_{j+1}^{k}\left(Y_{(j+1) i}\right), \ldots, I_{L}^{k}\left(Y_{L i}\right) ; F, N\right) \\
& \cdot \hat{L}\left(Y_{1 i}, Y_{2 i}, \ldots, Y_{L i}\right), \quad \text { for } i=1, \ldots, N, j=1, \ldots, L .
\end{aligned}
$$

Lemma 4.4. $C_{M C}\left(I_{1}^{k+1}, \ldots, I_{j}^{k+1}, I_{j+1}^{k}, \ldots, I_{L}^{k} ; F, N\right)$ is non-increasing as $j$ is increased and $C_{M C}\left(I_{1}^{k+1}\right.$, $\left.I_{2}^{k+1}, \ldots, I_{L}^{k+1} ; F, N\right) \leq C_{M C}\left(I_{1}^{k}, I_{2}^{k}, \ldots, I_{L}^{k} ; F, N\right)$.

Proof. Using (34)-(35), we have

$$
C_{M C}\left(I_{1}^{k+1}, \ldots, I_{j}^{k+1}, I_{j+1}^{k}, \ldots, I_{L}^{k} ; F, N\right)=\frac{1}{N} \sum_{i=1}^{N} \frac{\left[1-I_{j}^{k+1}\left(Y_{j i}\right)\right]}{g\left(Y_{1 i}, Y_{2 i}, \ldots, Y_{L i}\right)} G_{j}^{k+1}\left(Y_{j i}\right)+C_{j},
$$

where

$C_{j}=\frac{1}{N} \sum_{i=1}^{N} P_{j 2}\left(I_{1}^{k+1}\left(Y_{1 i}\right), \ldots, I_{j-1}^{k+1}\left(Y_{(j-1) i}\right), I_{j+1}^{k}\left(Y_{(j+1) i}\right), \ldots, I_{L}^{k}\left(Y_{L i}\right) ; F, N\right) \frac{\hat{L}\left(Y_{1 i}, Y_{2 i}, \ldots, Y_{L i}\right)}{g\left(Y_{1 i}, Y_{2 i}, \ldots, Y_{L i}\right)}+c$ is a constant independent of $I_{j}^{k}$ and $I_{j}^{k+1}$.

$$
\begin{aligned}
& C_{M C}\left(I_{1}^{k+1}, \ldots, I_{j}^{k+1}, I_{j+1}^{k}, \ldots, I_{L}^{k} ; F, N\right) \\
= & \frac{1}{N} \sum_{i=1}^{N} \frac{\left[1-I_{j}^{k}\left(Y_{j i}\right)\right]+\left[I_{j}^{k}\left(Y_{j i}\right)-I_{j}^{k+1}\left(Y_{j i}\right)\right]}{g\left(Y_{1 i}, Y_{2 i}, \ldots, Y_{L i}\right)} G_{j}^{k+1}\left(Y_{j i}\right)+C_{j} \\
= & \frac{1}{N} \sum_{i=1}^{N} \frac{\left[1-I_{j}^{k}\left(Y_{j i}\right)\right]}{g\left(Y_{1 i}, Y_{2 i}, \ldots, Y_{L i}\right)} G_{j}^{k+1}\left(Y_{j i}\right)+C_{j}+\frac{1}{N} \sum_{i=1}^{N} \frac{\left[I_{j}^{k}\left(Y_{j i}\right)-I_{j}^{k+1}\left(Y_{j i}\right)\right]}{g\left(Y_{1 i}, Y_{2 i}, \ldots, Y_{L i}\right)} G_{j}^{k+1}\left(Y_{j i}\right) \\
= & C_{M C}\left(I_{1}^{k+1}, \ldots, I_{j-1}^{k+1}, I_{j}^{k}, \ldots, I_{L}^{k} ; F, N\right)+D_{j}^{k+1},
\end{aligned}
$$

where

$$
D_{j}^{k+1}=\frac{1}{N} \sum_{i=1}^{N} \frac{\left[I_{j}^{k}\left(Y_{j i}\right)-I_{j}^{k+1}\left(Y_{j i}\right)\right] G_{j}^{k+1}\left(Y_{j i}\right)}{g\left(Y_{1 i}, Y_{2 i}, \ldots, Y_{L i}\right)}
$$


Note that (30)-(32) implie that $I_{j}^{k+1}\left(Y_{j i}\right)=0$ if and only if $G_{j}^{k+1}\left(Y_{j i}\right)<0$ and $I_{j}^{k+1}\left(Y_{j i}\right)=1$ if and only if $G_{j}^{k+1}\left(Y_{j i}\right) \geq 0$ for $i=1, \ldots, N, j=1, \ldots, L$. That is to say

$$
\left[I_{j}^{k}\left(Y_{j i}\right)-I_{j}^{k+1}\left(Y_{j i}\right)\right] G_{j}^{k+1}\left(Y_{j i}\right) \leq 0 .
$$

Thus, for $\forall i, j, k$

$$
\frac{\left[I_{j}^{k}\left(Y_{j i}\right)-I_{j}^{k+1}\left(Y_{j i}\right)\right] G_{j}^{k+1}\left(Y_{j i}\right)}{g\left(Y_{1 i}, Y_{2 i}, \ldots, Y_{L i}\right)} \leq 0
$$

the inequality holds because $g(\cdot)$ is a trial distribution and well-defined (i.e., $g(\cdot)>0$ ). Then the summation of all terms $D_{j}^{k+1} \leq 0$. Thus, for $\forall j \leq L, C_{M C}\left(I_{1}^{k+1}, \ldots, I_{j}^{k+1}, I_{j+1}^{k}, \ldots, I_{L}^{k} ; F, N\right) \leq C_{M C}\left(I_{1}^{k+1}, \ldots, I_{j-1}^{k+1}\right.$, $\left.I_{j}^{k}, \ldots, I_{L}^{k} ; F, N\right), C_{M C}\left(I_{1}^{k+1}, I_{2}^{k+1}, \ldots, I_{L}^{k+1} ; F, N\right) \leq C_{M C}\left(I_{1}^{k}, I_{2}^{k}, \ldots, I_{L}^{k} ; F, N\right)$.

Note that $C_{M C}\left(I_{1}^{k}, I_{2}^{k}, \ldots, I_{L}^{k} ; F, N\right)$ is finite valued. From Lemma 4.4 it must converge to a stationary point after a finite number of iterations.

Theorem 4.5. The $I_{1}^{k}, I_{2}^{k}, \ldots, I_{L}^{k}$ are finitely convergent.

Proof. By Lemma 4.4, $C_{M C}\left(I_{1}^{k}, I_{2}^{k}, \ldots, I_{L}^{k} ; F, N\right)$ must converge to a stationary point after a finite number of iterations, i. e.

$$
C_{M C}\left(I_{1}^{k+1}, \ldots, I_{j}^{k+1}, I_{j+1}^{k}, \ldots, I_{L}^{k} ; F, N\right)=C_{M C}\left(I_{1}^{k+1}, \ldots, I_{j-1}^{k+1}, I_{j}^{k}, \ldots, I_{L}^{k} ; F, N\right) .
$$

Using (36) and (40), we can derive that $D_{j}^{k+1}=0$. Combine (37)-(39),

$$
\left[I_{j}^{k}\left(Y_{j i}\right)-I_{j}^{k+1}\left(Y_{j i}\right)\right] G_{j}^{k+1}\left(Y_{j i}\right)=0 \quad \text { for } \quad i=1, \ldots, N,
$$

which implies either

$$
I_{j}^{k}\left(Y_{j i}\right)-I_{j}^{k+1}\left(Y_{j i}\right)=0, \quad \text { i.e. } \quad I_{j}^{k}\left(Y_{j i}\right)=I_{j}^{k+1}\left(Y_{j i}\right)
$$

or

$$
G_{j}^{k+1}\left(Y_{j i}\right)=0, \quad \text { i.e. } \quad I_{j}^{k+1}\left(Y_{j i}\right)=1 .
$$

It follows that when $C_{M C}$ converges to a stationary point, either $I_{j}^{k+1}\left(Y_{j i}\right)$ is invariant, or $I_{j}^{k+1}\left(Y_{j i}\right)=$ $1, I_{j}^{k}\left(Y_{j i}\right)=0$. That is $I_{j}^{k+1}\left(Y_{j i}\right)$ can only change from 0 to 1 at most a finite number of times. Thus the algorithm often cannot oscillate infinitely.

Theorem 4.6. For the fixed AND fusion rule, $\left(I_{1}\left(y_{1}\right), I_{2}\left(y_{2}\right), \ldots, I_{L}\left(y_{L}\right)\right)$ minimize the Monte Carlo cost function (17) if and only if they satisfy the following equations:

$$
\begin{aligned}
& I_{1}\left(Y_{1 i}\right) \cdot I_{2}\left(Y_{2 i}\right) \cdots I_{L}\left(Y_{L i}\right)=1 \text { if } \hat{L}\left(Y_{1 i}, Y_{2 i}, \ldots, Y_{L i}\right) \geq 0 \quad \text { for } \quad i=1, \cdots, N \\
& I_{1}\left(Y_{1 i}\right) \cdot I_{2}\left(Y_{2 i}\right) \cdots I_{L}\left(Y_{L i}\right)=0 \text { if } \hat{L}\left(Y_{1 i}, Y_{2 i}, \ldots, Y_{L i}\right)<0 \quad \text { for } \quad i=1, \cdots, N .
\end{aligned}
$$


Moreover, one of the optimal solutions is

$$
\begin{aligned}
& I_{1}\left(Y_{1 i}\right)=I_{2}\left(Y_{2 i}\right)=\cdots=I_{L}\left(Y_{L i}\right)=1 \text { if } \hat{L}\left(Y_{1 i}, Y_{2 i}, \ldots, Y_{L i}\right) \geq 0 \quad \text { for } \quad i=1, \cdots, N \\
& I_{1}\left(Y_{1 i}\right)=I_{2}\left(Y_{2 i}\right)=\cdots=I_{L}\left(Y_{L i}\right)=0 \text { if } \hat{L}\left(Y_{1 i}, Y_{2 i}, \ldots, Y_{L i}\right)<0 \quad \text { for } \quad i=1, \cdots, N
\end{aligned}
$$

Proof. For the fixed AND fusion rule,

$$
I_{\Omega_{0}}\left(Y_{1 i}, Y_{2 i}, \ldots, Y_{L i}\right)=1-I_{1}\left(Y_{1 i}\right) \cdot I_{2}\left(Y_{2 i}\right) \cdots I_{L}\left(Y_{L i}\right)
$$

Substituting (45) into (16) and simplifying, we have

$$
\begin{aligned}
& C_{M C}\left(I_{1}\left(y_{1}\right), \ldots, I_{L}\left(y_{L}\right) ; F, N\right) \\
= & \frac{1}{N} \sum_{i=1}^{N}\left\{1-I_{1}\left(Y_{1 i}\right) \cdot I_{2}\left(Y_{2 i}\right) \cdots I_{L}\left(Y_{L i}\right)\right\} \cdot \frac{\hat{L}\left(Y_{1 i}, Y_{2 i}, \ldots, Y_{L i}\right)}{g\left(Y_{1 i}, Y_{2 i}, \ldots, Y_{L i}\right)}+c \\
= & \frac{1}{N} \sum_{i=1}^{N} \frac{\hat{L}\left(Y_{1 i}, Y_{2 i}, \ldots, Y_{L i}\right)}{g\left(Y_{1 i}, Y_{2 i}, \ldots, Y_{L i}\right)}-\frac{1}{N} \sum_{i=1}^{N} I_{1}\left(Y_{1 i}\right) \cdot I_{2}\left(Y_{2 i}\right) \cdots I_{L}\left(Y_{L i}\right) \cdot \frac{\hat{L}\left(Y_{1 i}, Y_{2 i}, \ldots, Y_{L i}\right)}{g\left(Y_{1 i}, Y_{2 i}, \ldots, Y_{L i}\right)}+c \\
= & C_{0}-\tilde{C}_{M C}\left(I_{1}\left(y_{1}\right), \ldots, I_{L}\left(y_{L}\right) ; F, N\right)
\end{aligned}
$$

where

$$
\begin{aligned}
C_{0} & =\frac{1}{N} \sum_{i=1}^{N} \frac{\hat{L}\left(Y_{1 i}, Y_{2 i}, \ldots, Y_{L i}\right)}{g\left(Y_{1 i}, Y_{2 i}, \ldots, Y_{L i}\right)}+c \\
\tilde{C}_{M C}\left(I_{1}\left(y_{1}\right), \ldots, I_{L}\left(y_{L}\right) ; F, N\right) & =\frac{1}{N} \sum_{i=1}^{N} I_{1}\left(Y_{1 i}\right) \cdot I_{2}\left(Y_{2 i}\right) \cdots I_{L}\left(Y_{L i}\right) \cdot \frac{\hat{L}\left(Y_{1 i}, Y_{2 i}, \ldots, Y_{L i}\right)}{g\left(Y_{1 i}, Y_{2 i}, \ldots, Y_{L i}\right)} .(46)
\end{aligned}
$$

$C_{0}$ is a constant, then minimizing $C_{M C}\left(I_{1}\left(y_{1}\right), \ldots, I_{L}\left(y_{L}\right) ; F, N\right)$ is equivalent to maximize $\tilde{C}_{M C}\left(I_{1}\left(y_{1}\right)\right.$, $\left.\ldots, I_{L}\left(y_{L}\right) ; F, N\right)$. Note that $g\left(Y_{1 i}, Y_{2 i}, \ldots, Y_{L i}\right)>0$ and $I_{1}\left(Y_{1 i}\right) \cdot I_{2}\left(Y_{2 i}\right) \cdots I_{L}\left(Y_{L i}\right)=1$ or 0 . For arbitrary $Y_{i}=\left(Y_{1 i}, Y_{2 i}, \cdots, Y_{L i}\right),\left(I_{1}\left(y_{1}\right), I_{2}\left(y_{2}\right), \ldots, I_{L}\left(y_{L}\right)\right)$ maximize $\tilde{C}_{M C}$ if and only if they satisfy the following equations:

$$
\begin{aligned}
& I_{1}\left(Y_{1 i}\right) \cdot I_{2}\left(Y_{2 i}\right) \cdots I_{L}\left(Y_{L i}\right)=1 \text { if } \hat{L}\left(Y_{1 i}, Y_{2 i}, \ldots, Y_{L i}\right) \geq 0 \quad \text { for } \quad i=1, \cdots, N \\
& I_{1}\left(Y_{1 i}\right) \cdot I_{2}\left(Y_{2 i}\right) \cdots I_{L}\left(Y_{L i}\right)=0 \text { if } \hat{L}\left(Y_{1 i}, Y_{2 i}, \ldots, Y_{L i}\right)<0 \quad \text { for } \quad i=1, \cdots, N
\end{aligned}
$$

Thus, we have (41)- (42).

Theorem 4.7. For the fixed OR fusion rule, $\left(I_{1}\left(y_{1}\right), I_{2}\left(y_{2}\right), \ldots, I_{L}\left(y_{L}\right)\right)$ minimize the Monte Carlo cost function (17) if and only if they satisfy the following equations:

$$
\begin{aligned}
& \left(1-I_{1}\left(Y_{1 i}\right)\right) \cdot\left(1-I_{2}\left(Y_{2 i}\right)\right) \cdots\left(1-I_{L}\left(Y_{L i}\right)\right)=0 \text { if } \hat{L}\left(Y_{1 i}, Y_{2 i}, \ldots, Y_{L i}\right) \geq 0 \text { for } i=1, \cdots, N \\
& \left(1-I_{1}\left(Y_{1 i}\right)\right) \cdot\left(1-I_{2}\left(Y_{2 i}\right)\right) \cdots\left(1-I_{L}\left(Y_{L i}\right)\right)=1 \text { if } \hat{L}\left(Y_{1 i}, Y_{2 i}, \ldots, Y_{L i}\right)<0 \text { for } i=1, \cdots, N
\end{aligned}
$$


Moreover, one of the optimal solutions is

$$
\begin{aligned}
& I_{1}\left(Y_{1 i}\right)=I_{2}\left(Y_{2 i}\right)=\cdots=I_{L}\left(Y_{L i}\right)=1 \text { if } \hat{L}\left(Y_{1 i}, Y_{2 i}, \ldots, Y_{L i}\right) \geq 0 \quad \text { for } \quad i=1, \cdots, N \\
& I_{1}\left(Y_{1 i}\right)=I_{2}\left(Y_{2 i}\right)=\cdots=I_{L}\left(Y_{L i}\right)=0 \text { if } \hat{L}\left(Y_{1 i}, Y_{2 i}, \ldots, Y_{L i}\right)<0 \quad \text { for } \quad i=1, \cdots, N
\end{aligned}
$$

Proof. For the fixed OR fusion rule,

$$
I_{\Omega_{0}}\left(Y_{1 i}, Y_{2 i}, \ldots, Y_{L i}\right)=\left(1-I_{1}\left(Y_{1 i}\right)\right) \cdot\left(1-I_{2}\left(Y_{2 i}\right)\right) \cdots\left(1-I_{L}\left(Y_{L i}\right)\right)
$$

Substituting (51) into (16), we have

$$
\begin{aligned}
& C_{M C}\left(I_{1}\left(y_{1}\right), \ldots, I_{L}\left(y_{L}\right) ; F, N\right) \\
= & \frac{1}{N} \sum_{i=1}^{N}\left\{\left(1-I_{1}\left(Y_{1 i}\right)\right) \cdot\left(1-I_{2}\left(Y_{2 i}\right)\right) \cdots\left(1-I_{L}\left(Y_{L i}\right)\right)\right\} \cdot \frac{\hat{L}\left(Y_{1 i}, Y_{2 i}, \ldots, Y_{L i}\right)}{g\left(Y_{1 i}, Y_{2 i}, \ldots, Y_{L i}\right)}+c .
\end{aligned}
$$

Since $c$ is a constant, $\left(1-I_{1}\left(Y_{1 i}\right)\right) \cdot\left(1-I_{2}\left(Y_{2 i}\right)\right) \cdots\left(1-I_{L}\left(Y_{L i}\right)\right)=0$ or 1 and $g\left(Y_{1 i}, Y_{2 i}, \ldots, Y_{L i}\right)>0$, $\left(I_{1}\left(y_{1}\right), I_{2}\left(y_{2}\right), \ldots, I_{L}\left(y_{L}\right)\right)$ minimize $C_{M C}\left(I_{1}\left(y_{1}\right), \ldots, I_{L}\left(y_{L}\right) ; F, N\right)$ if and only if they satisfy the following equations:

$$
\begin{aligned}
& \left(1-I_{1}\left(Y_{1 i}\right)\right) \cdot\left(1-I_{2}\left(Y_{2 i}\right)\right) \cdots\left(1-I_{L}\left(Y_{L i}\right)\right)=0 \text { if } \hat{L}\left(Y_{1 i}, Y_{2 i}, \ldots, Y_{L i}\right) \geq 0 \text { for } i=1, \cdots, N \\
& \left(1-I_{1}\left(Y_{1 i}\right)\right) \cdot\left(1-I_{2}\left(Y_{2 i}\right)\right) \cdots\left(1-I_{L}\left(Y_{L i}\right)\right)=1 \text { if } \hat{L}\left(Y_{1 i}, Y_{2 i}, \ldots, Y_{L i}\right)<0 \text { for } i=1, \cdots, N
\end{aligned}
$$

Thus, we have (47)-(48).

Theorem 4.8. For the fixed AND fusion rule and any initial value, the solution of Monte Carlo Gauss-Seidel iterative algorithm must converge to the analytically optimal solution given in Theorem 4.6.

Proof. Without loss of generality, we assume that Monte Carlo Gauss-Seidel iterative algorithm terminated at $K$-th iteration for any initial value and $\left(I_{1}^{K}\left(Y_{1 i}\right), I_{2}^{K}\left(Y_{2 i}\right), \ldots, I_{L}^{K}\left(Y_{L i}\right)\right)$ is the set of $\mathrm{L}$ sensor decision rules at $K$-th iteration. We need to prove that

$$
\begin{aligned}
& I_{1}^{K}\left(Y_{1 i}\right) \cdot I_{2}^{K}\left(Y_{2 i}\right) \cdots I_{L}^{K}\left(Y_{L i}\right)=1 \quad \text { if } \quad \hat{L}\left(Y_{1 i}, Y_{2 i}, \ldots, Y_{L i}\right) \geq 0 \quad \text { for } \quad i=1, \cdots, N \\
& I_{1}^{K}\left(Y_{1 i}\right) \cdot I_{2}^{K}\left(Y_{2 i}\right) \cdots I_{L}^{K}\left(Y_{L i}\right)=0 \quad \text { if } \quad \hat{L}\left(Y_{1 i}, Y_{2 i}, \ldots, Y_{L i}\right)<0 \quad \text { for } \quad i=1, \cdots, N \text {. }
\end{aligned}
$$

Define two sets $S_{Y 1}$ and $S_{Y 0}$,

$$
\begin{aligned}
& S_{Y 1}=\left\{Y_{i} \mid \hat{L}\left(Y_{1 i}, Y_{2 i}, \ldots, Y_{L i}\right) \geq 0, i=1, \cdots, N\right\} \\
& S_{Y 0}=\left\{Y_{i} \mid \hat{L}\left(Y_{1 i}, Y_{2 i}, \ldots, Y_{L i}\right)<0, i=1, \cdots, N\right\} .
\end{aligned}
$$


Firstly, we prove (52) by a contradiction. If there exists a sample $Y_{m} \in S_{Y 1}$ such that $I_{1}^{K}\left(Y_{1 m}\right)$. $I_{2}^{K}\left(Y_{2 m}\right) \cdots I_{L}^{K}\left(Y_{L m}\right)=0$, which implies that there must exist some $j$ such that $I_{j}^{K}\left(Y_{j m}\right)=0$. For the fixed AND fusion rule, by (12), for $j=1, \ldots, L$

$$
\begin{aligned}
& P_{j 1}\left(I_{1}\left(Y_{1 m}\right), \ldots, I_{j-1}\left(Y_{(j-1) m}\right), I_{j+1}\left(Y_{(j+1) m}\right), \ldots, I_{L}\left(Y_{L m}\right)\right) \\
= & I_{1}\left(Y_{1 m}\right) \cdots I_{j-1}\left(Y_{(j-1) m}\right) \cdot I_{j+1}\left(Y_{(j+1) m}\right) \cdots I_{L}\left(Y_{L m}\right) .
\end{aligned}
$$

Thus, $P_{j 1}\left(I_{1}\left(Y_{1 m}\right), \ldots, I_{j-1}\left(Y_{(j-1) m}\right), I_{j+1}\left(Y_{(j+1) m}\right), \ldots, I_{L}\left(Y_{L m}\right)\right)=1$ or 0 for $j=1, \ldots, L$. Moreover, $P_{j 1}\left(I_{1}^{K+1}\left(Y_{1 m}\right), \ldots, I_{j-1}^{K+1}\left(Y_{(j-1) m}\right), I_{j+1}^{K}\left(Y_{(j+1) m}\right), \ldots, I_{L}^{K}\left(Y_{L m}\right)\right)=1$ or 0 for $j=1, \ldots, L$. We can conclude that $P_{j 1}\left(I_{1}^{K+1}\left(Y_{1 m}\right), \ldots, I_{j-1}^{K+1}\left(Y_{(j-1) m}\right), I_{j+1}^{K}\left(Y_{(j+1) m}\right), \ldots, I_{L}^{K}\left(Y_{L m}\right)\right) \cdot \hat{L}\left(Y_{1 m}, Y_{2 m}, \ldots\right.$, $\left.Y_{L m}\right) \geq 0$ because of $Y_{m} \in S_{Y 1}$, that is $\hat{L}\left(Y_{1 m}, Y_{2 m}, \ldots, Y_{L m}\right) \geq 0$. By (30)-(32), $I_{j}^{K+1}\left(Y_{j m}\right)=1 \neq$ $I_{j}^{K}\left(Y_{j m}\right)=0$. It is a contradiction. Thus, we have (52).

Secondly, we prove (53) by a contradiction. If there exists a sample $Y_{n} \in S_{Y 0}$ such that $I_{1}^{K}\left(Y_{1 n}\right)$. $I_{2}^{K}\left(Y_{2 n}\right) \cdots I_{L}^{K}\left(Y_{L n}\right)=1$, which implies that $I_{j}^{K}\left(Y_{j n}\right)=1$ for $j=1, \ldots, L$. By (54), we can conclude $P_{11}\left(I_{2}^{K}\left(Y_{2 n}\right), \ldots, I_{L}^{K}\left(Y_{L n}\right)\right)=1$. Since $Y_{n} \in S_{Y 0}$, that is $\hat{L}\left(Y_{1 n}, Y_{2 n}, \ldots, Y_{L n}\right)<0$. Thus,

$$
P_{11}\left(I_{2}^{K}\left(Y_{2 n}\right), \ldots, I_{L}^{K}\left(Y_{L n}\right)\right) \cdot \hat{L}\left(Y_{1 n}, Y_{2 n}, \ldots, Y_{L n}\right)<0
$$

By Algorithm 4.1 and (28), we can conclude that $I_{1}^{K+1}\left(Y_{1 n}\right)=0 \neq I_{1}^{K}\left(Y_{1 n}\right)=1$. It is a contradiction. Thus, we have (53).

Remark 4.9. Since the OR fusion rule and AND fusion rule are dual each other, for the fixed OR fusion rule and any initial value, the solution of Algorithm 4.1 must converge to the analytically optimal solution given in Theorem 4.7

\section{Numerical Examples}

To evaluate the performance of the new algorithm, we investigate some examples with large number of sensors where observation signal $s$ and observation noises are assumed Gaussian and independent. Thus, the observations are dependent. Since the previous distributed detection algorithm with general dependent observations does not work when the number of sensors is more than 5, we evaluate the new algorithm by comparing it with the centralized likelihood ratio method with 10 sensors and 100 sensors, respectively.

\subsection{Ten sensors}

We consider Monte Carlo importance sampling methods with AND, OR and 2 out of 5 (2/5) fusion rule.

Example 5.1. Let us consider ten sensors model with observation signal $s$ and observation noises $v_{1}, v_{2}$, 
$\ldots, v_{10}$,

$$
\begin{aligned}
& H_{1}: y_{i}=s+v_{i}, \text { for } i=1, \ldots, 10 \\
& H_{0}: y_{i}=v_{i}, \text { for } i=1, \ldots, 10
\end{aligned}
$$

where $s, v_{1}, v_{2} \ldots, v_{10}$ are all mutually independent and

$$
s \sim N(1,0.4), \quad v_{i} \sim N(0,0.6), \text { for } i=1, \ldots, 10
$$

Therefore, the two conditional pdfs given $H_{0}$ and $H_{1}$ are

$$
\begin{aligned}
p\left(y_{1}, y_{2}, \ldots, y_{10} \mid H_{1}\right) \sim N & \left(\left(\begin{array}{c}
1 \\
1 \\
\vdots \\
1
\end{array}\right),\left(\begin{array}{cccc}
1 & 0.4 & \cdots & 0.4 \\
0.4 & 1 & \cdots & 0.4 \\
\vdots & \vdots & \ddots & \vdots \\
0.4 & 0.4 & \cdots & 1
\end{array}\right)\right) \\
p\left(y_{1}, y_{2}, \ldots, y_{10} \mid H_{0}\right) \sim N & \left(\left(\begin{array}{c}
0 \\
0 \\
\vdots \\
0
\end{array}\right),\left(\begin{array}{cccc}
0.6 & 0 & \cdots & 0 \\
0 & 0.6 & \cdots & 0 \\
\vdots & \vdots & \ddots & \vdots \\
0 & 0 & \cdots & 0.6
\end{array}\right)\right)
\end{aligned}
$$

In Figure 1, the ROC curves for Centralized algorithm, Algorithm 4.1 with a mixture Gaussian trial distribution and Algorithm 4.1 with a Gaussian trial distribution are provided where the AND, OR and 2 out of 5 (2/5) fusion rules are considered, respectively. For Algorithm 4.1, we draw $N=1000$ samples from the trial distribution. The initial values of the sensor rule are $I_{i}\left(y_{i}\right)=I\left[3 y_{i}-4\right]$, for $i=1, \cdots, L$.

The solid line is the ROC curve calculated by the centralized algorithm. The star line, circle line and square line are the ROC curves for the fixed AND, OR and 2 out of 5 (2/5) fusion rule calculated by Algorithm 4.1 with Mixture-Gaussian trial distribution, respectively. The $\times$ line, diamond line and $*$ line line are the ROC curves for the fixed AND, OR and 2 out of 5 (2/5) fusion rule calculated by Algorithm 4.1 with Gaussian trial distribution, respectively.

From Figure 1, we have the following observations:

- The performance of Algorithm 4.1 with Mixture-Gaussian trial distribution is better than that of Algorithm 4.1 with Gaussian trial distribution. The reason may be that the optimal trial distribution in (16) should be $g\left(y_{1}, y_{2}, \ldots, y_{L}\right) \propto\left|I_{\Omega_{0}}\left(y_{1}, y_{2}, \ldots, y_{L}\right) \hat{L}\left(y_{1}, y_{2}, \ldots, y_{L}\right)\right|$ (see, e.g., [25, 26]) and $\left|\hat{L}\left(y_{1}, y_{2}, \ldots, y_{L}\right)\right|=\left|a p\left(y_{1}, \ldots, y_{L} \mid H_{1}\right)-b p\left(y_{1}, \ldots, y_{L} \mid H_{0}\right)\right|$ which is similar to Mixture-Gaussian. Thus, the performance based on Mixture-Gaussian trial distribution is better than that of Gaussian trial distribution.

- When probability of a false alarm $P_{f}$ is small, the performance of the fixed AND fusion rule is better than that of the fixed OR fusion rule and vice versa. 


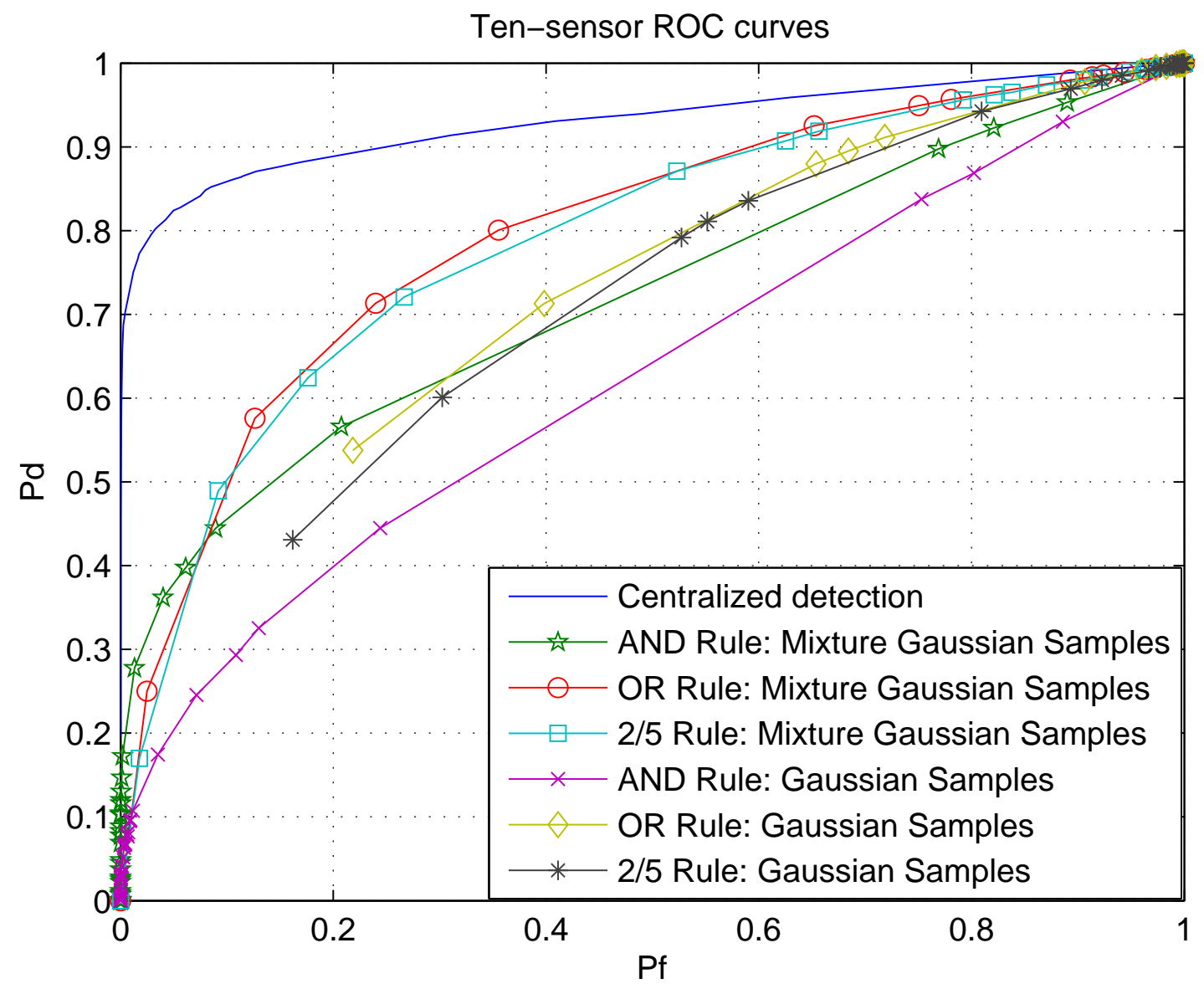

Figure 1: Ten-sensor ROC curves.

- For the same parameters, most of points of the AND fusion rule converge to the $(0,0)$ and most of points of the OR fusion rule converge to the $(1,1)$. The reason may be the AND fusion rule corresponds to a smaller probability of a false alarm $P_{f}$ than that of the OR fusion rule.

\subsection{One hundred sensors}

Example 5.2. Let us consider a surveillance model. There is a target/signal s which may cross a surveillance region from one of 50 paths with an equal probability. 100 sensors are deployed on the 50 paths separately. Each path has two sensors. The 100 sensors transmit the decision 0 or 1 to the fusion center. We consider a given fusion rule that if there is only one path where two sensors make a decision $(1,1)$, then the fusion center makes a decision 1; otherwise, make a decision 0. 
The signal $s$ and observation noises $v_{1}, v_{2}, \ldots, v_{100}$ are all mutually independent and

$$
s \sim N(1,0.4), \quad v_{i} \sim N(0,0.6), \text { for } i=1, \ldots, 100
$$

Thus, the two conditional probability density functions ( $p d f s$ ) given $H_{0}$ and $H_{1}$ are

$$
\begin{aligned}
& p\left(y_{1}, y_{2}, \ldots, y_{100} \mid H_{0}\right) \sim N\left(\mu_{0}, \Sigma_{0}\right), \\
& p\left(y_{1}, y_{2}, \ldots, y_{100} \mid H_{1}\right) \sim \sum_{i=1}^{50} P \times N\left(\mu_{1, i}, \Sigma_{1, i}\right),
\end{aligned}
$$

where $P=1 / 50$,

$$
\begin{aligned}
& \mu_{0}=(0, \cdots, 0)_{100 \times 1}^{\prime}, \quad \Sigma_{0}=\operatorname{diag}(0.6, \cdots, 0.6)_{100 \times 100}, \\
& \mu_{1,1}=\left(\mu^{\prime}, 0, \cdots, 0\right)_{100 \times 1}^{\prime}, \cdots, \mu_{1,50}=\left(0, \cdots, 0, \mu^{\prime}\right)_{100 \times 1}^{\prime}, \\
& \Sigma_{1,1}=\operatorname{diag}(\Sigma, 0.6, \cdots, 0.6)_{100 \times 100}, \cdots, \Sigma_{1,50}=\operatorname{diag}(0.6, \cdots, 0.6, \Sigma)_{100 \times 100}, \\
& \mu=\left(\begin{array}{l}
1 \\
1
\end{array}\right)_{2 \times 1}, \Sigma=\left(\begin{array}{cc}
1 & 0.4 \\
0.4 & 1
\end{array}\right)_{2 \times 2} .
\end{aligned}
$$

In Figure 2, the ROC curves for Centralized algorithm, Algorithm 4.1 with a mixture Gaussian trial distribution and Algorithm 4.1 with a Gaussian trial distribution are provided. For Algorithm 4.1, we draw $N=10000$ samples from the trial distribution to derive the optimal sensor decision rules.

The solid line is the ROC curve calculated by the centralized algorithm. The circle line is the ROC curve for the fixed fusion rule by Algorithm 4.1 with Mixture-Gaussian trial distribution. The star line is the ROC curve for the fixed fusion rule calculated by with Gaussian trial distribution.

From Figure 2, it can be seen that the performance of Algorithm 4.1 with Mixture-Gaussian trial distribution is better than that of Algorithm 4.1 with Gaussian trial distribution. The reason is similar to the case of two sensors or ten sensors. This example also shows that the new method can be applied to large number of sensor networks when the fusion rule is fixed.

\section{Conclusion}

In the paper, we have proposed a Monte Carlo framework for the distributed detection fusion with highdimension conditionally dependent observations. By using the Monte Carlo importance sampling, we derived a necessary condition for optimal sensor decision rules so that a Gauss-Seidel optimization approach can be obtained to search the optimal sensor decision rules. We proved that the discretized algorithm is finitely convergent. The complexity of the new algorithm is order of $O(L N)$ compared with $O\left(L N^{L}\right)$ of the previous

algorithm where $L$ is the number of sensors and $N$ is the sample size in the importance sampling draw. Thus, the proposed methods allows us to design the large sensor networks with general dependent observations. 


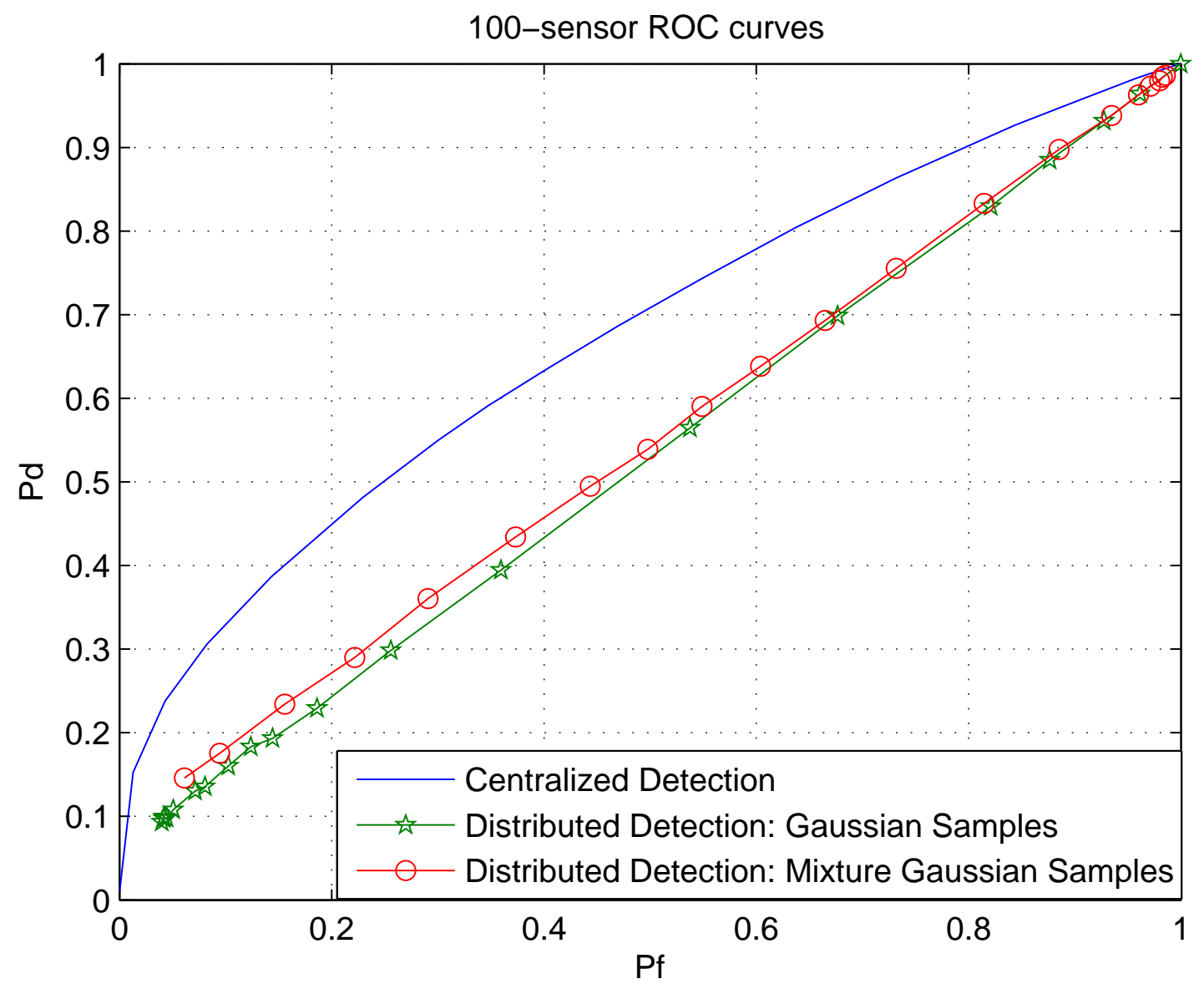

Figure 2: One-hundred-sensor ROC curves

Furthermore, an interesting result is that, for the fixed AND or OR fusion rules, we have analytically derived the optimal solution in the sense of minimizing the approximated Bayesian cost function. In general, the solution of the Gauss-Seidel algorithm is only local optimal. However, in the new framework, we have proved that the solution of Gauss-Seidel algorithm is same as the analytically optimal solution in the case of the AND or OR fusion rule. The typical examples with dependent observations and large number of sensors are examined under this new framework. The results of numerical examples demonstrate the effectiveness of the new algorithm class. Future work will involve the generalization of the Monte Carlo framework for parallel networks to all kinds of networks. When the number of the sensors is very large, how to optimize the fusion rule is another challenging problem. 


\section{References}

[1] R. R. Tenney and N. R. Sandell, "Detection with distributed sensors," IEEE Transaction on Aerospace and Electronic Systems, vol. 17, no. 4, pp. 501-510, 1981.

[2] J. N. Tsitsiklis and M. Athans, "On the complexity of decentralized decision making and detection problems," IEEE Transactions on Automatic Control, vol. 30, pp. 440-446, 1985.

[3] Z. Chair and P. K. Varshney, "Optimal data fusion in multiple sensor detection systems," IEEE Transaction on Aerospace and Electronic Systems, vol. 22, pp. 98-101, January 1986.

[4] P. K. Varshney, Distributed Detection and Data Fusion. New York: Springer-Verlag, 1997.

[5] R. S. Blum, S. A. Kassam, and H. V. Poor, "Distributed detection with multiple sensors: Part IIcadvanced topics," Proceedings of the IEEE, vol. 85, pp. 64-79, 1997.

[6] B. Chen and P. Willett, "On the optimality of the likelihood-ratio test for local sensor decision rules in the presence of nonideal channels," IEEE Transactions on Information Theory, vol. 52, no. 2, pp. 693699., 2005.

[7] B. Chen, L. Tong, and P. K. Varshney, "Channel-aware distributed detection in wireless sensor networks," IEEE Signal Processing Magazine, vol. 23, no. 4, pp. 16-26, 2006.

[8] Y. Zhu, J. Zhou, X. Shen, E. Song, and Y. Luo, Networked Multisensor Decision and Estimation Fusion: Based on Advanced Mathematical Methods. CRC Press, 2012.

[9] A. Kashyap, "Comments on the optimally of the likelihood-ratio test for local sensor decision rules in the presence of nonideal channel," IEEE Transactions on Information Theory, vol. 52, no. 2, pp. 67-72, 2006.

[10] H. Chen, B. Chen, and P. K. Varshney, "Further results on the optimality of likelihood ratio quantizer for distributed detection in nonideal channels," IEEE Transactions on Information Theory, vol. 55, pp. 828-832, February 2009.

[11] I. Y. Hoballah and P. K. Varshney, "Distributed Bayesian signal detection," IEEE Transactions on Information Theory, vol. 35, pp. 995-1000, 1989.

[12] E. Drakopoulos and C. C. Lee, "Optimum multisensor fusion of correlated local decisions," IEEE Transactions on Aerospace and Electronic Systems, vol. 27, pp. 424-429, 1991.

[13] I. Kam, Q. Zhu, W. S. Gray, and W. Steven, "Optimal data fusion of correlated local decisions in multiple sensor detection systems," IEEE Transaction on Aerospace and Electronic Systems, vol. 28, pp. 916-920, July 1992. 
[14] R. S. Blum and S. A. Kassam, "Optimum-distributed detection of weak signals in dependent sensors," IEEE Transactions on Information Theory, vol. 36, pp. 1066-1079, 1992.

[15] Z. B. Tang, K. R. Pattipati, and D. L. Kleinman, "A distributed M-ary hypothesis testing problem with correlated observations," IEEE Transactions on Automatic Control, vol. 37, pp. 1042-1046, July 1992.

[16] P. N. Chen and A. Papamarcou, "Likelihood ratio partitions for distributed signal detection in correlated gaussian noise," Proceedings of IEEE International sympathesis Information Theory, p. 118, October 1995.

[17] Q. Yan and R. S. Blum, "Distributed signal detection under the neyman-pearson criterion," IEEE Transactions on Information Theory, vol. 47, no. 4, pp. 1368-1377, 2001.

[18] P. Willett, P. F. Swaszek, and R. S. Blum, "The good, bad and ugly: Distributed detection of a known signal in dependent Gaussian noise," IEEE Transactions on Signal Processing, vol. 48, pp. 3266-3279, December 2000.

[19] Y. Zhu, R. S. Blum, Z.-Q. Luo, and K. M. Wong, "Unexpected properties and optimum-distributed sensor detectors for dependent observation cases," IEEE Transactions on Automatic Control, vol. 45, pp. 62-72, January 2000.

[20] X. Shen, Y. Zhu, L. He, and Z. You, "A near-optimal iterative algorithm via alternately optimizing sensor and fusion rules in distributed decision systems," IEEE Transactions on Aerospace and Electronic Systems, vol. 47, pp. 2514-2529, October 2011.

[21] H. Chen, B. Chen, and P. K. Varshney, "A new framework for distributed detection with conditionally dependent observations," IEEE Transactions on Signal Processing, vol. 60, pp. 1409-1419, March 2012.

[22] J. N. Tsitsiklis, "Decentralized detection by a large number of sensors," Mathematics of Control, Signals and Systems, vol. 1, no. 2, pp. 167-182, 1988.

[23] J.-F. Chamberland and V. V. Veeravalli, "Decentralized detection in sensor networks," IEEE Transactions on Signal Processing, vol. 51, no. 2, pp. 407-416, 2003.

[24] R. Niu and P. K. Varshney, "Distributed detection and fusion in a large wireless sensor network of random size," EURASIP Journal on Wireless Communications and Networking, vol. 2005, pp. 462472, 42005.

[25] J. S. Liu, Monte Carlo Strategies in Scientific Computing. New York: Springer, 2001.

[26] R. Christian and C. George, Monte Carlo Statistical Methods. Springer Science \& Business Media, 2005. 